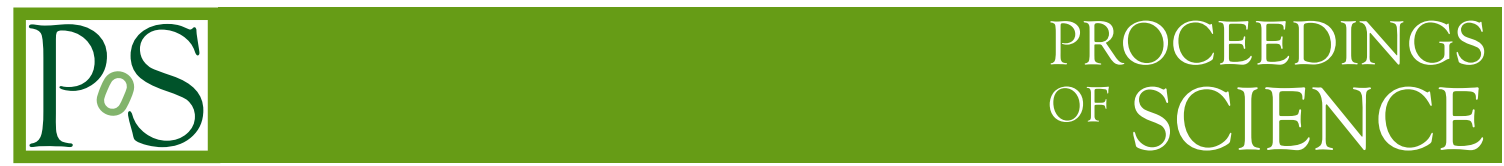

\title{
The Phase Diagram of QCD Matter
}

\author{
N.G. Antoniou* \\ Faculty of Physics, University of Athens, Greece \\ E-mail: nantonio@phys.uoa.gr
}

Three outstanding phenomena along the critical line in the QCD phase diagram are discussed: (a) the crossover transition at zero chemical potential, (b) the critical fluctuations at the QCD critical point and (c) the development of viscosity in the net-baryon fluid, near the critical temperature. Signatures of these phenomena in high-energy experiments with nuclei, are also discussed.

Corfu Summer Institute 2016 "School and Workshops on Elementary Particle Physics and Gravity" 31 August - 23 September, 2016

Corfu, Greece

${ }^{*}$ Speaker. 


\section{The crossover transition}

The properties of strongly interacting matter can in principle be revealed within the context of lattice QCD, first principle calculations, based on a Euclidean Lagrangian with physical quark masses $m$ and a gauge coupling $g$ [1].

$$
\begin{gathered}
\mathscr{L}=\frac{1}{2 g^{2}} \operatorname{Tr}\left(F_{\mu \nu} F_{\mu v}\right)+\bar{\psi} \gamma_{\mu}\left(\partial_{\mu}+A_{\mu}+m\right) \psi \\
F_{\mu v}=\partial_{\mu} A_{v}-\partial_{v} A_{\mu}+\left[A_{\mu}, A_{v}\right]
\end{gathered}
$$

The transition from QCD Lagrangian (eq. 1.1) to QCD Thermodynamics is achieved with the help of the action $S$ which leads to the partition function $Z$ of the system at high temperatures (where the quark-hadron transition occurs) and zero chemical potential:

$$
S=\int d^{4} x \mathscr{L}, \quad Z=\sum \exp (-S)
$$

The ambiguities in lattice QCD studies are removed if we use physical quark masses and extrapolate to vanishing lattice spacing (continuum limit). After enormous theoretical and computational effort, these requirements are now fulfilled and the lattice formulation (1.2) is well defined at zero chemical potential $\left(\mu_{b}=0\right)$. A serious problem still remains, for $\mu_{b} \neq 0$, and this difficulty prohibits the unambiguous, first-principle description of strongly interacting matter near the QCD critical point. The chemical potential issue arises from the integration of fermion fields in eq. (1.2) which leads to an effective theory containing only bosonic fields (gluons):

$$
Z=\int[\mathscr{D} v] \operatorname{det} M(v) \exp \left[-S_{g}(v)\right]
$$

The measure of integration in eq. (1.3) is positive definite for $\mu_{b}=0$ but at non-vanishing chemical potentials the fermion determinant can take complex values and the probabilistic interpretation of eq. (1.3) is no longer valid. Several proposals to overcome this difficulty exist but a general consensus is still missing [2].

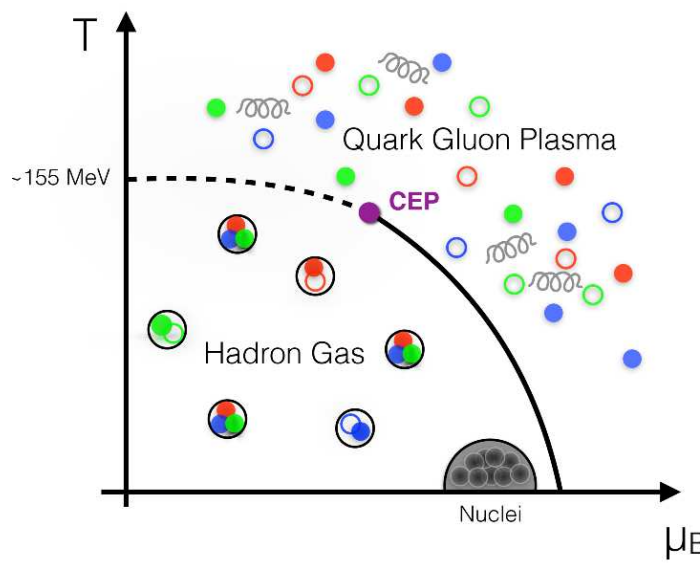

Figure 1: Phase diagram of QCD. Crossover is depicted with slashed line.
Within this context, the nature of the QCD transition at $\mu_{b}=0$ can be studied, without ambiguities, leading to a rigorous result: The phenomenon of quark-hadron phase transition in this corner of the phase diagram (Fig. 1) is an analytical crossover. The proof is based on the study of finite-size scaling at the susceptibility peak which occurs when the system approaches the critical temperature $T=T_{c}$. The behaviour of the volume dependence is drastically different if the transition is of first or second order, or if 
it is an analytical crossover:

$$
\begin{aligned}
& \text { 1st order transition : } \chi_{T}^{\max } \sim V \\
& \text { 2nd order transition : } \chi_{T}^{\max } \sim V^{\gamma / v d}(\gamma, v \text { : critical exponents) } \\
& \text { crossover transition : } \chi_{T}^{\max } \sim \text { const. }
\end{aligned}
$$

In general, the susceptibility of a thermal system is obtained from the second derivative of the Gibbs free energy $G$ with respect to the ordering field $H$ [3]:

$$
G=-T \ln Z, M=-\frac{\partial G}{\partial H}, \chi=\frac{1}{V} \frac{\partial M}{\partial H} \rightarrow \chi=\frac{\partial^{2}}{\partial H^{2}}\left(\frac{T}{V} \ln Z\right)
$$

For chiral susceptibility, the ordering field is the mass of light quarks $m_{u, d}$, therefore:

$$
\chi_{c h}\left(N_{s}, N_{t}\right)=\frac{\partial^{2}}{\partial m_{u, d}^{2}}\left(\frac{T}{V} \ln Z\right)
$$

where $N_{s}\left(N_{t}\right)$ is the spatial (Euclidean time) extension.

A remarkable result is given in reference [1] where a study of $\chi_{c h}$ is performed in a lattice with two choices of spatial extension: $4 \times 12^{3}, 4 \times 24^{3}$ corresponding to a volume ratio $\frac{V_{2}}{V_{1}}=8$. According to the rule (1.4) a first-order phase transition would predict a susceptibility peak, $\chi_{c h}^{\max }$, eight times higher for the second choice of spatial extension, whereas for a second-order phase transition this ratio would be approximately four (corresponding to the $3 \mathrm{~d}$ Ising universality class, $v \simeq \frac{2}{3}, \gamma \simeq \frac{4}{3}$ ). The computational solution [1] shows clearly that there is no volume dependence, providing us with a proof that the phenomenon of quark-hadron transition at $\mu_{b}=0$ is an analytical crossover. The result is exact and suggests that the crossover phenomenon can be extended along the critical line $\left(\mu_{b} \neq 0\right)$ with a limiting location at the QCD critical point where the transition becomes of second order. This conjecture remains to be shown together with the solution of the finite $\mu_{b}$ problem in lattice QCD.

Finally, it is of interest to note that the QCD transition for small values of the baryonic density, takes place in a cosmological path and therefore the nature of the phase transition may affect the detailed mechanism of Big Bang nucleosynthesis. In fact, a strong first-order phase transition creates baryon rich nuggets which could remain for a long time, contributing to dark matter. Theses inhomogeneities in the hadronic phase could have a strong effect on nucleosynthesis [4]. Obviously, in a physical world where the Universe has undergone a crossover transition, the above picture, based on a strong first-order transition, is ruled out.

Experimental support to the transition being a crossover at small $\mu_{b}$ can only be indirect. Using the crossover equation of state in a hydrodynamic description of charged hadrons, produced in high-energy nuclear collisions, the data on invariant yields at LHC are easily explained [5]. Moreover, the ratio of baryon susceptibilities of various orders, given by lattice calculations with a crossover at $\mu_{b}=0$ [2] agree with experimental measurements of similar quantities, extracted from correlations of protons, on the basis of fluctuation-dissipation relations.

\section{Critical fluctuations}

The QCD critical point at high temperatures $\left(T_{c} \simeq 200 \mathrm{MeV}\right)$ is a distinct phenomenon. It is the endpoint of a series of first-order phase transitions which occur at relatively high values of the 
chemical potential $\frac{\mu_{b}}{T_{c}}>1$. A first principle theory of the QCD critical point is not yet available, mainly due to the ambiguities of lattice QCD formulation at $\mu_{b} \neq 0$. However, owing to the fact that at the critical endpoint (CEP) the system of strongly interacting matter undergoes a second-order phase transition, the universality principle may reveal significant properties of the critical system which do not depend on the microscopic details of the theory. In this context one may try to build a universal, effective theory with the following tools and ingredients, linked to the macroscopic, critical profile of the system:

1) The order parameter: It is linked to an extensive thermodynamic variable accessible to measurements. Near the critical point the order parameter describes, with its behaviour, the fundamental property of symmetry breaking and it is, in this region, the only important thermodynamic quantity. In the case of QCD critical point, a natural choice for the order parameter is the chiral condensate $\langle\bar{\psi} \psi\rangle$ with the quantum numbers of a scalar-isoscalar field (sigma field). In a finitedensity medium, the critical fluctuations of the sigma field develop the same singular behaviour as the baryon-number density $n_{b}\left(\delta \sigma \simeq \delta n_{b}\right)$ and therefore, $n_{b}$ is also qualified as an order parameter of the QCD critical system [6]. In fact, if we consider the requirements of dynamics, $n_{b}$ is the only unambiguous order parameter since, as a conserved quantity, is a "slow" thermodynamic variable whereas the $\sigma$-field, being massive near the critical point, represents a "fast" variable during the dynamical process of relaxation [7]. This fundamental distinction follows from the behaviour of long wavelength components of $n_{b}$ and $\sigma$ :

(a) the conservation law of baryon number leads to the continuity equation in Fourier space:

$$
\frac{\partial n_{b}(\vec{k}, t)}{\partial t}+i \vec{k} \cdot \vec{j}(\vec{k}, t)=0
$$

where $\vec{k}$ is the wave-number vector. In the long wavelength limit $(k \rightarrow 0)$ the motion of the corresponding components $n_{b}(\vec{k}, t)$ is very slow and this property guarantees a correct behaviour of $n_{b}$ as an order parameter,

(b) on the contrary, a massive $\sigma$-field leads to a dispersion relation, $\omega=\left(k^{2}+m_{\sigma}^{2}\right)^{1 / 2}$ corresponding to a fast mode of the long wavelength components $(k \rightarrow 0)$. Only in the chiral limit $\left(m_{\sigma}=0\right)$ the long wavelength components correspond to a slow mode, $\omega \sim k$, and the chiral condensate ( $\sigma$-field) becomes an unambiguous order parameter of chiral phase transition.

2) The universal effective action: The QCD critical point belongs to the $3 \mathrm{~d}$ Ising universality class with approximate critical exponents: $\alpha \simeq 0, \beta \simeq \frac{1}{3}, \gamma \simeq \frac{4}{3}, \delta \simeq 5, v \simeq \frac{2}{3}$ and $\eta=0$. In this framework, a universal effective action was found recently [8] on the basis of a Monte Carlo computation in the $3 \mathrm{~d}$ Ising model, in an external field. Introducing a dimensionless scalar field $\phi=\beta_{c}^{3} n_{b}$ as the order parameter, compatible with our previous discussion, the Tsypin effective action [8] for $T=T_{c}$ is written as follows:

$$
S_{e f f}=\int d^{3} \hat{x}\left[\frac{1}{2}|\hat{\nabla} \phi|^{2}+G \phi^{\delta+1}-\hat{H} \phi\right]
$$

where $\hat{x}_{i}=\frac{x_{i}}{\beta_{c}}$ and $\hat{H}=\left(\mu-\mu_{c}\right) \beta_{c}$, the ordering field, identified with appropriate (dimensionless) chemical-potential variable of the net-baryon fluid.

On the basis of the discussion above and the formulation (2.2) one may extract the baryon density fluctuations at the critical point $\left(T=T_{c}, \mu=\mu_{c}\right)$ and try to make them accessible to 
measurements. Considering the ensemble of constant configurations $\phi$ in the partition function $Z=\sum_{\phi} \exp \left(-S_{e f f}\right)$ we obtain a finite-size scaling relation for the average multiplicity $\left\langle N_{b}\right\rangle$ as a function of volume: $\left\langle N_{b}\right\rangle \sim V^{5 / 6}$. This behaviour suggests a fractal geometry of the produced clusters of baryons, with fractal dimension $d_{F}=\frac{5}{2}$. This index is a measure of the critical fluctuations developed at the critical point and it is directly linked to the isothermal critical exponent $\delta$ : $d_{F}=\frac{\delta d}{\delta+1}$ [9]. In order these fluctuations to become accessible to experiment, a transformation of the fractal geometry from configuration to momentum space is required. In a relativistic process this can only be possible for the projection in transverse space where a Fourier transform is at work [10]. In transverse configuration space, the fractal dimension of the projected structure is $d_{F}^{(2)}=\frac{5}{3}$ and in transverse momentum space, the Fourier transform gives $\hat{d}_{F}^{(2)}=\frac{1}{3}$. This geometry implies density-density correlations of the form

$$
\left\langle n_{b}\left(\vec{k}_{\perp}\right) n_{b}\left(\vec{k}_{\perp}^{\prime}\right)\right\rangle \sim\left|\vec{k}_{\perp}-\vec{k}_{\perp}^{\prime}\right|^{-5 / 3}
$$

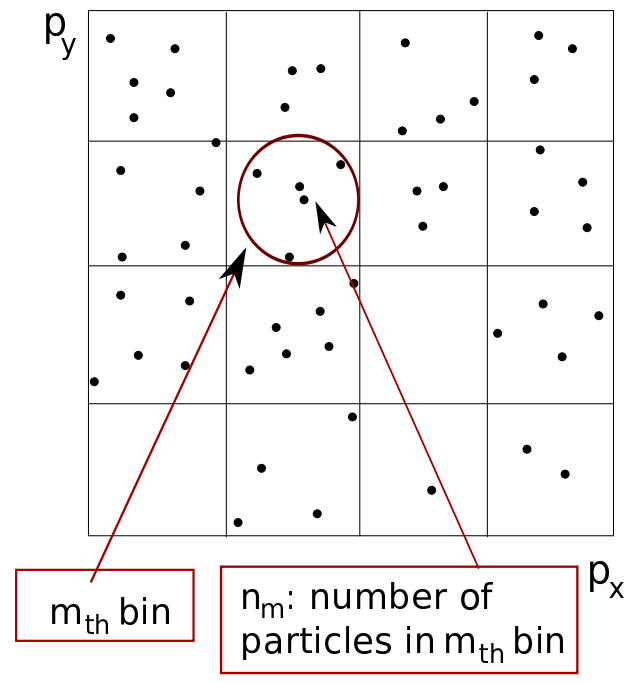

Figure 2: $2 \mathrm{~d}$ intermittency

revealing strong, local fluctuations of baryon density with a singular behaviour at small scales $\left(\left|\delta \vec{k}_{\perp}\right| \rightarrow 0\right)$. These power law fluctuations give rise to an intermittency phenomenon [11] detectable in high-energy processes, in particular in nuclear collisions where critical phenomena of strongly interacting matter are expected to occur [12]. The observables related to correlations (2.3) are factorial moments in $2 \mathrm{~d}$ (Fig. 2) computed in small cells, in the central region [13]. More precisely, the transverse momentum space is partitioned into $M^{2}$ cells and the factorial moments $F_{2}(M)$ are calculated as a function of cell size (or of the number of one dimensional cells $M$ ). The phenomenon of intermittency associated with the QCD critical point manifests itself as a power law of $F_{2}(M)$ for large $M$, as follows:

$$
F_{2}(M)=\frac{\sum_{m}\left\langle N_{m}\left(N_{m}-1\right)\right\rangle}{\sum_{m}\left\langle N_{m}\right\rangle^{2}} ; \quad F_{2}(M) \sim\left(M^{2}\right)^{\varphi_{2}}, \quad M \gg 1
$$

where $\varphi_{2}=\frac{5}{6}$. It is of interest to note that critical intermittency, as described above, is the analogue of critical opalescence in conventional matter [13]. In practice, in order to eliminate the effect of a strong background of uncorrelated baryons, the power law (2.4) must be applied to a properly defined correlator:

$$
\Delta F_{2}(M)=F_{2}^{\text {data }}(M)-F_{2}^{\text {mix }}(M) ; \Delta F_{2} \sim\left(M^{2}\right)^{5 / 6}
$$

where from the factorial moments $F_{2}^{\text {data }}$, computed using the events of actual data, we have subtracted a term corresponding to mixed events. The method of critical intermittency was applied to measurements of the experiment CERN-NA49 in a search for critical fluctuations of proton density 

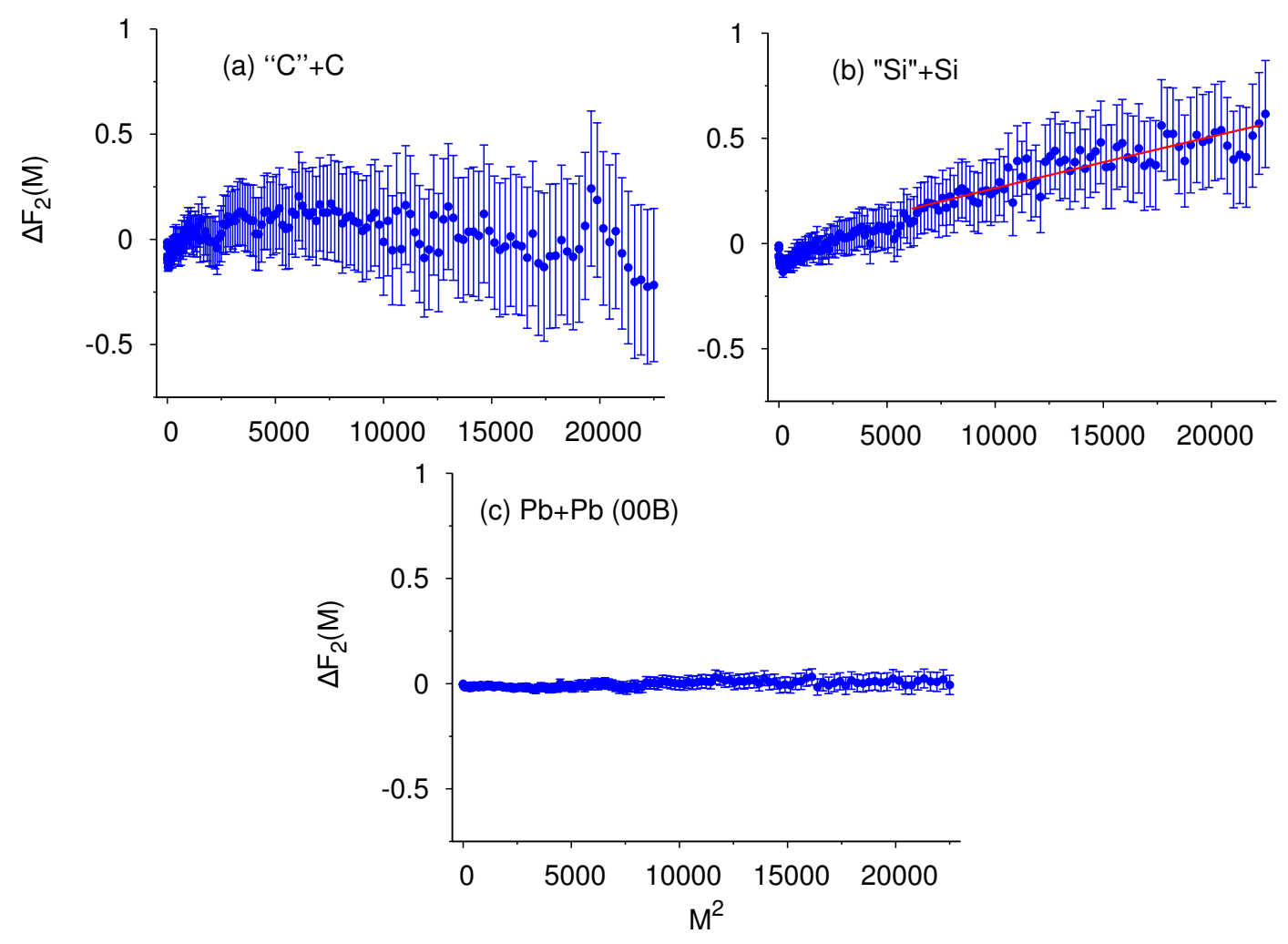

Figure 3: The correlator $\Delta F_{2}$ for proton pairs in SPS processes.

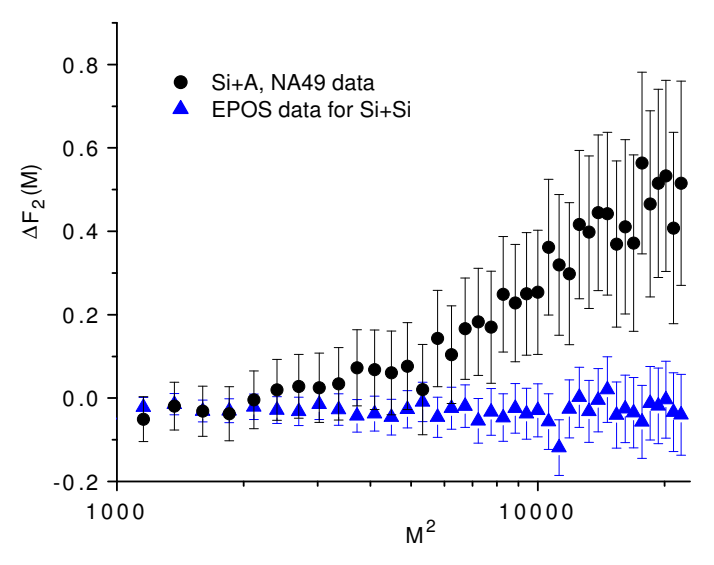

Figure 4: The correlator $\Delta F_{2}$ for proton pairs in Si-data, compared to EPOS simulation. in the processes $\mathrm{C}+\mathrm{C}, \mathrm{Si}+\mathrm{Si}, \mathrm{Pb}+\mathrm{Pb}$ at the highest CERN-SPS energy of $160 \mathrm{AGeV}$ [14]. The results for the correlator $\Delta F_{2}$ are given in Fig. 3 . A clear effect is observed in $\mathrm{Si}+\mathrm{Si}$ where the fitted value of the exponent $\varphi=0.96_{-0.25}^{+0.38}$ is compatible with the Ising-QCD value $\varphi_{2}^{\mathrm{QCD}}=\frac{5}{6}$ [13]. As a result, a strong indication was found for the existence of the QCD CEP and its location close to the freeze-out state in $\mathrm{Si}+\mathrm{Si}$ collisions at $\sqrt{s_{N N}}=17.3 \mathrm{GeV}: T \simeq 160 \mathrm{MeV}, \mu_{b} \simeq 260$ $\mathrm{MeV}$. No traces of critical fluctuations were found in the freeze-out states of $\mathrm{C}+\mathrm{C}$ and $\mathrm{Pb}+\mathrm{Pb}$, at the same energy. A further support to these findings comes from the comparison of measured correlator in $\mathrm{Si}+\mathrm{Si}$ (Fig. 4) with the output from EPOS event generator. In this simulation, all conventional final states are taken into account including energetic hadronic jets which could generate spurious intermittency by non-critical protons. It was found that EPOS events [14] cannot account for critical intermittency, leading to a correlator $\Delta F_{2}$ which, as a function of $M$, fluctuates around zero (Fig. 4).

In conclusion, the investigation above suggests that a dedicated experimental program of high 
precision with nuclei of size close to $\operatorname{Si}(A \simeq 30)$ and colliding energies close to SPS ( $\sqrt{s_{N N}} \simeq 17$ $\mathrm{GeV}$ ) may lead to the discovery of the QCD critical point. In fact, towards this line of research, the NA61 experiment at CERN-SPS [15] and the Beam Energy Scan Program at BNL-RHIC [16] are in progress.

\section{Viscosity of baryon-number fluid near $T_{c}$}

In the second part of this presentation (section 2) we have introduced the baryon number density $n_{b}$ as a proper order parameter, compatible with the long-time scale of the QCD critical phenomena [7]. As a consequence, the baryon-number fluid is appropriate to capture the dynamical aspects of the QCD critical point, in particular the development of singular viscosity (shear and bulk) near the critical temperature $T_{c}$. For the description of these phenomena we assume that, in a process out of equilibrium, net baryons relax to a $3 \mathrm{~d}$ Ising-like system in equilibrium. The basic mechanism, in this process, consists of thermal diffusion and sound waves. In this framework, one may consider the approximation in which, approaching the critical point during relaxation, the following thermodynamic quantities prevail in the description of shear $(\eta)$ and bulk $(\zeta)$ viscosity:

$$
\eta\left(T, v_{s}, \xi, \frac{c_{P}}{c_{V}}\right) ; \zeta\left(\rho, v_{s}, \xi, \frac{c_{P}}{c_{V}}\right)
$$

where $v_{s}$ is the velocity of sound waves, $\xi$ : the correlation length and $\rho$ : the mass density of the medium in the bulk. On the basis of dimensional considerations: [viscosity] $=[$ energy density] $\times$ [time] one may obtain the following expressions in terms of singular quantities in the limit $T \rightarrow T_{c}$, $\mu_{b}=\mu_{c}$ :

$$
\frac{\eta}{s}=\frac{k_{B} T v_{s}^{-1}}{s \xi^{2}} F^{(s)}\left(\frac{c_{P}}{c_{V}}\right) ; \frac{\zeta}{s}=\frac{\rho v_{s} \xi}{s} F^{(b)}\left(\frac{c_{P}}{c_{V}}\right)
$$

where we have introduced the entropy density $(s)$ forming the dimensionless ratios (3.2) in the system of units $k_{B}=c=\hbar=1$. The basic thermodynamics of the fluid is formulated as follows:

$$
\begin{gathered}
c_{P}-c_{V}=T k_{T}\left(\frac{\partial P}{\partial T}\right)_{V}^{2}, \frac{c_{P}}{c_{V}}=\frac{k_{T}}{k_{S}}, v_{s}^{2}=\left(\rho k_{S}\right)^{-1}, \\
s=\frac{\varepsilon+P}{T}-\frac{\mu_{b} n_{b}}{T}, \rho=\frac{\varepsilon+P}{c^{2}}
\end{gathered}
$$

where $k_{T}, k_{S}$ are the isothermal and adiabatic (isoentropic) compressibility, $\varepsilon$ : the energy density, $P$ : the pressure and $\mu_{b}$ : the baryochemical potential [17].

The development of singular viscosity near the critical point describes the dynamical properties of the fluid [7] along the critical line (Fig. 1) whereas, in a distance from the critical temperature, in the quark phase $\left(T \gg T_{c}\right)$, we meet the equation of state of an ideal, massless, classical system:

$$
\begin{gathered}
\varepsilon=3 P, P=n_{b} T, h=4 n_{b} T \text { (h: enthalpy density) }, c_{V}=3 n_{b}, \\
c_{P}=4 n_{b}, v_{s}=\frac{1}{\sqrt{3}}, k_{T}=\left(n_{b} T\right)^{-1}, s=\left(4-\frac{\mu_{b}}{T}\right) n_{b}
\end{gathered}
$$

An analytical solution for the viscosity of the baryon-number fluid is expected to provide us with a smooth transition from the critical region of singular behaviour $\left(T \geq T_{c}\right)$ to the ideal system 
(3.4) at high temperatures $\left(T \gg T_{c}\right)$. To this end we consider the power laws, valid in the limit $T \rightarrow T_{c}, \mu_{b}=\mu_{c}$ :

$$
c_{V}=A_{ \pm}|t|^{-\alpha}, k_{T}=\Gamma_{ \pm}|t|^{-\gamma}, \xi=\xi_{ \pm}|t|^{-v}\left(t \equiv \frac{T-T_{c}}{T_{c}}\right)
$$

where the indices $\alpha, \gamma, v$ are the $3 \mathrm{~d}$ Ising-like critical exponents and the amplitudes $A_{ \pm}, \Gamma_{ \pm}, \xi_{ \pm}$ correspond to the limits $t \rightarrow 0^{+}$and $t \rightarrow 0^{-}$respectively. The ratios of the amplitudes $\frac{A_{+}}{A_{-}}, \frac{\Gamma_{+}}{\Gamma_{-}}, \frac{\xi_{+}}{\xi_{-}}$ are also universal and are fixed within the universality class of the critical point [18].

In this context, a treatment of shear and bulk viscosity near the QCD critical point can be performed on the basis of eqs. $(3.2,3.3,3.5)$. For the functions $F^{(i)}\left(\frac{c_{P}}{c_{V}}\right), i:(s, b)$ in eqs. (3.2) we adopt a simple description inspired by a perturbative treatment of conventional fluids in the vicinity of liquid-gas critical point [19]: $F^{(i)}=f^{(i)} \frac{c_{P}}{c_{V}}$ where $f^{(i)}$ are dimensionless, nonuniversal constants. In order to specify the solution for $(\eta, \zeta)$ the following constraints are in order:

(a) The ratios of the amplitudes are fixed by the $3 \mathrm{~d}$ Ising universality class [20]: $\frac{A_{+}}{A_{-}} \simeq 0.55, \frac{\xi_{+}}{\xi_{-}} \simeq 2$, $\frac{\Gamma_{+}}{\Gamma_{-}} \simeq 4.5$ and

(b) The solution for $T>T_{c}$ meets the ideal system (3.4) at $T=2 T_{c}$ where the sound velocity reaches the value $v_{s}=\frac{1}{\sqrt{3}}$. The boundary condition at this point gives $A_{+}=3 n_{c}$ and $\Gamma_{+}=\left(2 n_{c} T_{c}\right)^{-1}$.

With these constraints, one obtains the following solution:

$$
\begin{gathered}
\left(\frac{\eta}{s}\right)_{ \pm}=f^{(s)} M_{ \pm}\left(1+\Lambda_{ \pm}|t|^{\gamma+3 v-2}\right)^{1 / 2}|t|^{1-\gamma+\frac{v}{2}} \\
\left(\frac{\zeta}{s}\right)_{ \pm}=f^{(b)} N_{ \pm}\left(1+\Lambda_{ \pm}|t|^{\gamma+3 v-2}\right)^{3 / 2}|t|^{3-\gamma-\frac{11}{2} v} \\
v_{s}^{2}=\frac{t^{2-3 v}}{4}\left(\frac{2 t^{\gamma+3 v-2}}{1+t}+\frac{1}{3}\right) ; T_{c} \leq T \leq 2 T_{c},\left\langle v_{s}^{2}\right\rangle=0.27
\end{gathered}
$$

where:

$$
\begin{gathered}
M_{+}=\frac{1}{\sqrt{3}} \frac{\xi_{+}^{-2} T_{c}}{s_{c}}, N_{+}=\frac{1}{3 \sqrt{3}} \frac{n_{c} \xi_{+} T_{c}}{s_{c}}, \Lambda_{+}=6 \\
M_{-}=\frac{4}{7 \sqrt{3}} \frac{\xi_{+}^{-2} T_{c}}{s_{c}}, N_{-}=\frac{1}{84 \sqrt{3}} \frac{n_{c} \xi_{+} T_{c}}{s_{c}}, \Lambda_{-}=60
\end{gathered}
$$

In writing the exponents in eqs. (3.6) we have used the Josephson scaling law $v d=2-\alpha$ [3] restricting the number of critical exponents involved to a couple of independent indices $(\gamma, v)$. From eqs. (3.6) the power laws in the limit $T \rightarrow T_{c}$ are for $T>T_{c}$ :

$$
\eta \sim|t|^{1-\gamma+\frac{v}{2}}, \zeta \sim|t|^{3-\gamma-\frac{11}{2} v}
$$

The behaviour (3.8) we require to be in accordance with the dynamical aspects of the QCD critical point and in particular with the prediction in reference [7] which is based on a RG treatment of an appropriate Langevin equation linked to these phenomena: $\eta \sim t^{-0.053 v}, \zeta \sim t^{-2.8 v}$. Comparing this prediction with the behaviour (3.8) we find: $\gamma=1.34, v=0.61$, a solution compatible with the Ising universality class in $3 \mathrm{~d}$ [18].

From eqs. $(3.6,3.7)$ we observe that the solution (3.6) depends on two collective parameters: $f^{(s)} \frac{\xi_{+}^{-2} T_{c}}{s_{c}}, f^{(b)} \frac{n_{c} \xi_{+} T_{c}}{s_{c}}$. For a set of indicative critical values: $T_{c} \simeq 160 \mathrm{MeV}, \mu_{c} \simeq 220 \mathrm{MeV}, n_{c} \simeq 0.13$ 


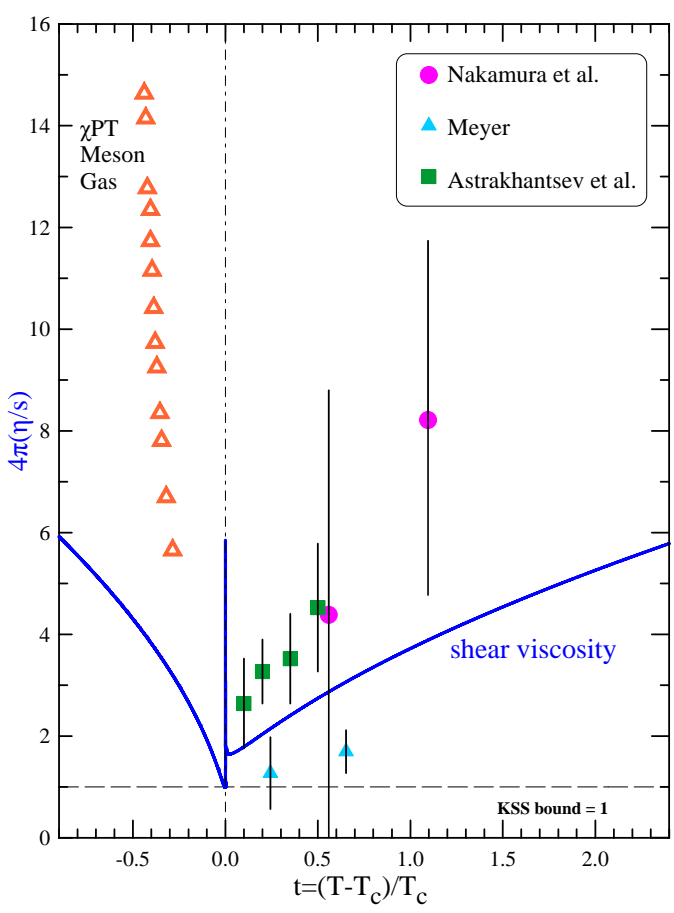

Figure 5: Our solution for the shear viscosity (blue line) compared with the findings of [25] (empty orange triangles), [26] (solid circles, triangles and squares).

$\mathrm{fm}^{-3}$ [21] and $\xi_{+} \simeq 1 \mathrm{fm}$, we end up in a description with the dimensionless constants $f^{(s)}$, $f^{(b)}$ which depend on the nature of the medium (net-baryon fluid). To complete our treatment and determine these parameters, we employ, as a final guiding principle, the KSS bound [22] which is assumed to be reacked by the minimum of the ratio $\frac{\eta}{s}$, close to the critical temperature. This constraint originates from a class of strong coupling field theories (AdS/CFT limit) and it is widely accepted that the formation of quark matter in high-energy nuclear collisions creates an ideal environment to test its validity [23]. In the same framework, a constraint on the bulk viscosity is obtained if we use the parametrization, $\frac{\zeta}{s}=\frac{1}{8 \pi}\left(\frac{1}{3}-v_{s}^{2}\right)$, introduced in reference [24] and take, for our purpose, the average in the domain $T_{c} \leq T \leq 2 T_{c}$ with the help of eqs. (3.6) in which $\left\langle v_{s}^{2}\right\rangle \simeq 0.27$. Thus, we obtain the final constraints:

$$
\begin{gathered}
\left(\frac{\eta}{s}\right)_{\min }=\frac{1}{4 \pi}\left(t \simeq 0^{-}\right) ; \\
\left\langle\left(\frac{\zeta}{s}\right)_{+}\right\rangle \simeq \frac{0.030}{4 \pi}
\end{gathered}
$$

which lead to the estimate: $f^{(s)} \simeq 8.2 \times 10^{-2}$ and $f^{(b)} \simeq 2.0 \times 10^{-3}$.

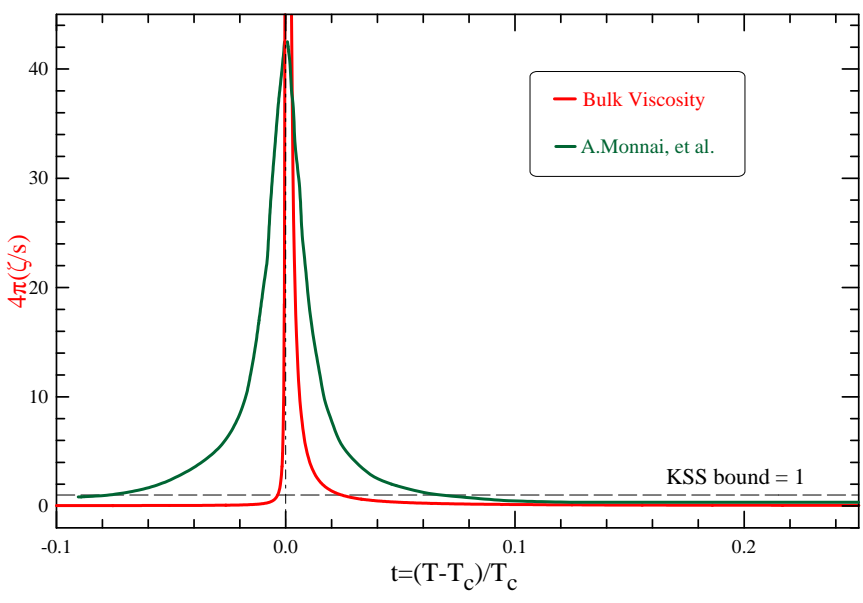

Figure 6: Our solution for the bulk viscosity (red line) compared with the findings of [27] (green line).

In Fig. 5 the solution for the shear viscosity of net-baryon matter is compared with other findings. In the hadronic phase $(T<$ $T_{c}$ ) the solution gives $1 \leq 4 \pi \frac{\eta}{s} \leq$ 4.3 for $0.5 T_{c} \leq T<T_{c}$, deviating from the behaviour of chiral matter [25]. For $T>T_{c}$ (quark matter phase) the result is $1.6 \leq 4 \pi \frac{\eta}{s} \leq$ 3.7 for $T_{c}<T \leq 2 T_{c}$ and a comparison with recent findings of lattice QCD [26] is illustrated. The weakness of the singularity at $T=T_{c}$ leads to a structure of two local minima, very close to the critical temperature (Fig. 5). The absolute minimum reaches the KSS bound, in the hadronic phase. This structure cannot be seen in the coarse data of the experiments and therefore is not expected to be observable in high-energy nuclear collisions. 
In Fig. 6 the solution for the bulk viscosity, eq. (3.6), of net-baryon matter is presented. It develops a strong divergent singularity at the critical point $\left(\zeta \sim \xi^{2.8}\right)$ but it decreases rapidly and stays to a constant value, smaller than the KSS bound, for $t \gg 0.025$. The result is compared with the solution in reference [27] where a dynamical treatment of enhanced bulk viscosity near the critical point is performed.

Concluding, in this last part of the presentation, we have described an analytical treatment of viscosity of the net-baryon matter, near the QCD critical point [28]. The approach was based on general issues, in particular on the aspects of the process of relaxation towards the critical state, on universality linked to criticality, on dynamical aspects of critical singularities and also on a constraint imposed by strong coupling theories (KSS bound).

In summary, we have discussed a number of distinct phenomena along the critical line, in the QCD phase diagram:

(a) The crossover transition at zero baryochemical potential $\left(\mu_{b}=0\right)$, an exact result of first principle calculations in lattice QCD.

(b) The development of critical fluctuations, compatible with QCD-Ising universality class, observed at SPS energies, in the freeze-out state of the process $\mathrm{Si}+\mathrm{Si}$ at $158 \mathrm{AGeV}$, and

(c) The development of singular viscosity of net-baryon matter, near the critical temperature. The size of shear viscosity, in the hadronic phase, was found in the domain $1 \leq 4 \pi \frac{\eta}{s} \leq 4.3$, in the temperature region $0.5 T_{c} \leq T \leq T_{c}$.

Together with the above results, a window of expectations is opened for the next few years. In particular, we expect:

(1) Final lattice QCD calculations at nonzero baryon-number density $\left(\mu_{b} \neq 0\right)$ leading to the location of the critical point (without ambiguities). Also, a calculation of transport coefficients (viscosity) with fully dynamical, light quarks.

(2) Precision measurements (NA61 experiment) in a search for critical fluctuations in collisions of light nuclei $(\mathrm{Be}+\mathrm{Be}, \mathrm{Ar}+\mathrm{S}, \mathrm{Xe}+\mathrm{La})$ at SPS energies. Also at RHIC in the Beam Energy Scan Program (BES), and

(3) Precision measurements of shear viscosity in net-baryon systems (elliptic flow of net protons + hydrodynamics) at SPS energies, close to the critical point.

\section{References}

[1] Y. Aoki, G. Endrődi, Z. Fodor, S.D. Katz, K.K. Szabó, Nature 443, 675 (2006).

[2] H-T. Ding, F. Karsch, S. Mukherjee, arXiv 1504.05274 [hep-lat].

[3] K. Huang, "Statistical Mechanics", Wiley, New York (1987).

[4] J.H. Applegate, C.J. Horgan, Phys. Rev. D 31, 3077 (1985).

[5] B. Mohanty, EPJ Web of Conferences 66, 04022 (2014); C. Gale et al. Phys. Rev. Lett. 110, 012302 (2013).

[6] K. Fukushima, T. Hatsuda, Rept. Prog. Phys. 74, 014001 (2011).

[7] Y. Minami, Phys. Rev. D 83, 094019 (2011).

[8] M.M. Tsypin, Phys. Rev. Lett. 73, 2015 (1994). 
[9] N.G. Antoniou, Y.F. Contoyiannis, F.K. Diakonos, C.G. Papadopoulos, Phys. Rev. Lett. 81, 4289 (1998).

[10] N.G. Antoniou, N. Davis, F. K. Diakonos, Phys. Rev. C 93, 014908 (2016).

[11] A. Bialas, R. Peshanski, Nucl. Phys. B 273, 703 (1986); 308, 857 (1988).

[12] T. Anticic et al. (NA49 experiment), Phys. Rev. C 81, 064907 (2010).

[13] N.G. Antoniou, F.K. Diakonos, A.S. Kapoyannis, K.S. Kousouris, Phys. Rev. Lett. 97, 032002 (2006).

[14] T. Anticic et al. (NA49 experiment), Eur. Phys. J. C 75, 587 (2015).

[15] N.G. Antoniou et al. CERN-SPSC-2006-34/p-330 and Addenda.

[16] STAR White Paper, Studying the phase diagram of QCD matter at RHIC (2014).

[17] N.G. Antoniou, F.K. Diakonos, A.S. Kapoyannis, arXiv: 1610.02028 (nucl-th) 2016.

[18] P.M. Chaikin, T.C. Lubensky, Principles of condensed matter physics, Cambridge University Press (1995).

[19] L.P. Kadanoff, J. Swift, Phys. Rev. 165, 310 (1968); 166, 89 (1968).

[20] V. Privmam, P.C. Hohenberg, A. Aharony, Phase Transitions and Critical Phenomena, Vol. 14, Eds. C. Domb and J.L. Lebowitz (Academic Press, New York, 1989).

[21] N.G. Antoniou, F.K. Diakonos, A.S. Kapoyannis, Phys. Rev. C 81, 011901 (R) (2010) .

[22] P.K. Kovtun, D.T. Son, A.O. Starinets, Phys. Rev. Lett. 94, 111601 (2005).

[23] L.P. Csernai, J.I. Kapusta, L.D. McLerran, Phys. Rev. Lett. 97, 152303 (2006).

[24] J. Noronha-Hostler et al., Phys. Rev. C 88, 044916 (2013); A. Buchel, Phys. Lett. B 663, 286 (2008).

[25] M. Prakash et al., Phys. Rept. 227, 321 (1993); J.-W. Chen et al., Phys. Rev. D 76, 114011 (2007).

[26] A. Nakamura, S. Sakai, Phys. Rev. Lett. 94, 072305 (2005); H. B. Meyer, Phys. Rev. D 76, 101701 (R) (2007); N.Yu. Astrakhantsev, V.V. Braguta, A.Yu. Kotov, arXiv 1701.02266 [hep-lat].

[27] A. Monnai, S. Mukherjee, Y. Yin, arXiv: 1606.00771 [nucl-th] (2016).

[28] N.G. Antoniou, F.K. Diakonos, A.S. Kapoyannis, arXiv: 1610.02028 [nucl-th]. 